\title{
In Situ Liquid Cell TEM/STEM with the Nanoaquarium
}

\author{
J. M. Grogan, H. H. Bau
}

Department of Mechanical Engineering and Applied Mechanics, University of Pennsylvania, 220 South $33^{\text {rd }}$ Street, Philadelphia, PA 19104

The last few years have seen a flare of efforts to develop devices that allow real-time, in situ imaging of dynamical, nanoscale processes in fluid media with the resolution of a TEM or STEM (scanning TEM). Liquid cell TEM/STEM devices confine a thin slice of liquid sample in a sealed chamber sandwiched between two electron-transparent membranes, thus preventing evaporation while allowing the electron beam to pass through the sample to produce an image. The liquid slice must be hermetically sealed from the vacuum chamber of the electron microscope and sufficiently thin to minimize electron scattering by the suspending medium.

Here, we present a new nanofluidic platform for in situ liquid cell TEM / STEM, dubbed the nanoaquarium (Figure 1). The nanoaquarium consists of a thin imaging liquid compartment, which can range in thickness from tens to hundreds of nanometers, sandwiched between two thin silicon nitride membranes that are transparent to electrons. The fabrication of the thin, hermetically sealed device is accomplished using direct wafer bonding of silicon wafers coated with silicon nitride [1]. One of the wafers also contains a thin film of patterned silicon oxide that defines the geometry and height of the chamber and conduits. The device featured in Figure 1 has a $100 \mathrm{~nm}$ thick silicon oxide film and two $50 \mathrm{~nm}$ thick imaging silicon nitride membranes. The wafer bonding approach reduces the possibility of contamination from glue, epoxy, or other sealing materials that are used in other devices. Use of a dielectric material as the spacer allows direct integration of electrodes on the device. The wafer-based batch microfabrication approach enables concurrent fabrication of many devices at a time (hundreds or thousands) with high reproducibility, high yield, and relatively low cost per unit. For example, Figure 2 shows a wafer with multiple devices patterned on it. The device fits into a custom-made holder and can sustain the high vacuum environment of the electron microscope for many hours without any noticeable loss of liquid. Some of the nanoaquarium's highlights include: an exceptionally thin sample cross-section; wafer scale processing that enables high yield mass production; robust hermetic sealing that provides leak-free operation; compatibility with lab-on-chip technology; and on-chip integrated electrodes for sensing and actuation.

The nanoaquarium has broad applicability for in situ electron microscope imaging of diverse nanoscale phenomena in liquid media that include nanoscale particle interactions; colloidal crystals [2] and formation of meta-materials with unique properties; electrochemical deposition and etching; processes associated with charging and discharging of batteries; interfacial phenomena; biological interactions; and protein and nanoparticle migration on lipid membranes. Here, we will discuss results of studies on the aggregation of gold nanoparticles and behavior of gold nanoparticles in a drying liquid film [3]. 


\section{References}

[1] J. M. Grogan and H. H. Bau, Journal of Microelectromechanical Systems. 19 (2010) 885.

[2] J. M. Grogan, L. Rotkina, and H. H. Bau, In Review.

[3] This work was supported in part by the NSF-NIRT (CBET 0609062), NSF-NBIC (NSF NSEC DMR-0425780), and the Nanotechnology Institute of Ben Franklin Technology Partners of Southeastern Pennsylvania. Device fabrication was carried out at the Cornell NanoScale Facility, a member of the NNIN, NSF (Grant ECS-0335765). Electron microscopy was performed at the Penn Regional Nanotechnology Facility. The aid of Dr. Lolita Rotkina, Dr. Frances M. Ross, Mr. Sumant Sood, and Mr. Peter Szczesniak is gratefully acknowledged.
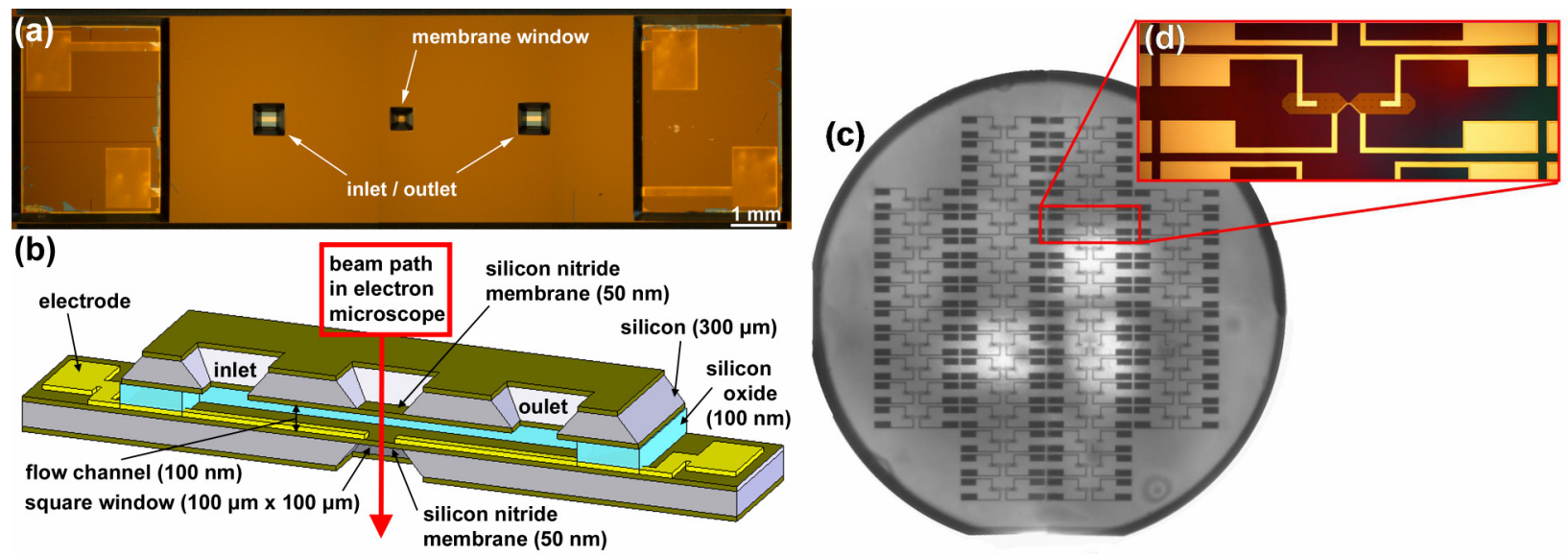

FIG. 1: The nanoaquarium. (a) Top-view photograph featuring the silicon nitride observation window and inlet/outlet ports. (b) A schematic of the cross-section. (c) A grayscale infrared image of a bonded pair of wafers with embedded nanoaquarium devices showing excellent void-free bonding. Each set of bonded wafers contains 52 devices. (d) Insert is a photo of the patterned bottom wafer prior to bonding.

FIG. 2: Bright field STEM image of an aqueous solution containing $5 \mathrm{~nm}$ gold particles. Insert is a zoomed in image taken 10 seconds later showing aggregate evolution.

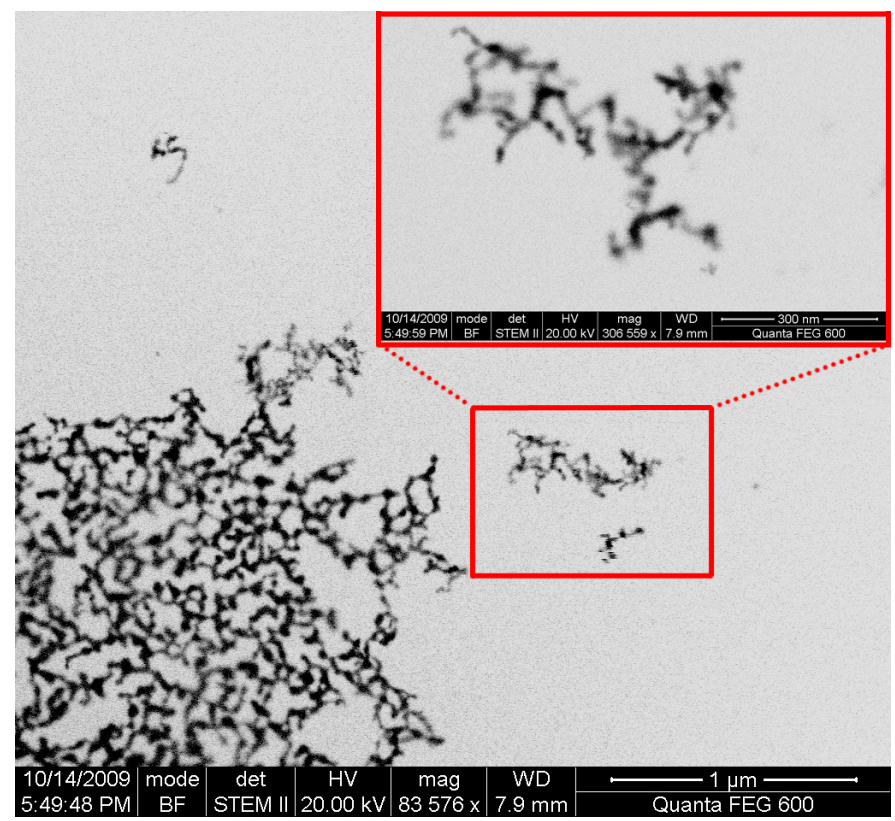

